\title{
Reversible-equivariant systems and matricial equations
}

\author{
MARCO A. TEIXEIRA and RICARDO M. MARTINS \\ Departamento de Matemática, Instituto de Matemática, Estatística e Computação Científica, \\ Universidade Estadual de Campinas/UNICAMP, Rua Sérgio Buarque de Holanda, 651 \\ Cidade Universitária, 13083-859 Campinas, SP, Brasil
}

Manuscript received on November 5, 2009; accepted for publication on August 13, 2010

\begin{abstract}
This paper uses tools in group theory and symbolic computing to classify the representations of finite groups with order lower than, or equal to 9 that can be derived from the study of local reversible-equivariant vector fields in $\mathbb{R}^{4}$. The results are obtained by solving matricial equations. In particular, we exhibit the involutions used in a local study of reversible-equivariant vector fields. Based on such approach we present, for each element in this class, a simplified Belitskii normal form.
\end{abstract}

Key words: Reversible-equivariant dynamical systems, involutory symmetries, normal forms.

\section{INTRODUCTION}

The presence of involutory symmetries and involutory reversing symmetries is very common in physical systems, for example, in classical mechanics, quantum mechanics and thermodynamic (see Lamb and Roberts 1996). The theory of ordinary differential equations with symmetry dates back from 1915 with the work of Birkhoff. Birkhoff realized a special property of his model: the existence of a involutive map $R$ such that the system was symmetric with respect to the set of fixed points of $R$. Since then, the work on differential equations with symmetries stay restricted to hamiltonian equations. Only in 1976, Devaney developed a theory for reversible dynamical systems.

In this paper, involutory symmetries and involutory reversing symmetries are considered within a unified approach. We study some possible linearizations for symmetries and reversing symmetries, around a fixed point, and employ this to simplify normal forms for a class of vector fields.

In particular, using tools from group theory and symbolic computing, we exhibit the involutions used in a local study of reversible-equivariant vector fields. Based on such approach we present, for each element in this class, a simplified Belitskii normal form. This new normal form simplifies the study of qualitative dynamics, unfoldings, and bifurcations, as we have to deal with a smaller number of parameters.

AMS Classification: 34C20, 37C80, 15A24.

Correspondence to: Ricardo Miranda Martins

E-mail: rmiranda@ime.unicamp.br 
An important point to mention is that any map possessing an involutory reversing symmetry is the composition of two involutions, as was found by Birkhoff in 1915. It is worth pointing out that properties of reversing symmetry groups are a powerful tool to study local bifurcation theory in presence of symmetries, see for instance Knus et al. (1998).

The authors are grateful to the referees for many helpful comments and suggestions.

\section{STATEMENT OF MAIN RESULTS}

Let $\mathfrak{X}_{0}\left(\mathbb{R}^{4}\right)$ denote the set of all germs of $C^{\infty}$ vector fields in $\mathbb{R}^{4}$ with an isolated singularity at origin. Define

$$
A(\alpha, \beta)=\left(\begin{array}{cccc}
0 & -\alpha & 0 & 0 \\
\alpha & 0 & 0 & 0 \\
0 & 0 & 0 & -\beta \\
0 & 0 & \beta & 0
\end{array}\right),
$$

with $\alpha, \beta \in \mathbb{R}, \alpha \beta \neq 0, \alpha \neq \beta$, and

$$
\mathfrak{X}_{0}^{(\alpha, \beta)}\left(\mathbb{R}^{4}\right)=\left\{X \in \mathfrak{X}_{0}\left(\mathbb{R}^{4}\right) ; D X(0)=A(\alpha, \beta)\right\} .
$$

The condition $\alpha \beta \neq 0$ is to keep the problem nondegenerate, while the condition $\alpha \neq \beta$ is necessary to avoid the appearence of 1-parameter families of symmetries (when working with reversible-equivariant vector field).

Given a group $G$ generated by involutive diffeomorphisms $\phi: \mathbb{R}^{4}, 0 \rightarrow \mathbb{R}^{4}, 0$ (involutive means $\left.\phi^{2}=I d\right)$ and a group homomorphism $\rho: G \rightarrow\{-1,1\}$, we say that $X \in \mathfrak{X}_{0}\left(\mathbb{R}^{4}\right)$ is $G$-reversibleequivariant if, for each $\phi \in G$,

$$
D \phi(x) X(x)=\rho(\phi) X(\phi(x)) .
$$

If $K \subseteq G$ is such that $\rho(K)=1$ we say that $X$ is $K$-equivariant. If $K \subset G$ is such that $\rho(K)=-1$, we say that $X$ is $K$-reversible. It is clear that if $X$ is $\phi_{1}$-reversible and $\phi_{2}$-reversible, then $X$ is also $\phi_{1} \phi_{2}$-equivariant. It is usual to denote $G_{+}=\{\phi \in G ; \rho(\phi)=1\}$ and $G_{-}=\{\phi \in G ; \rho(\phi)=-1\}$. Note that $G_{+}$is a subgroup of $G$, but $G_{-}$is not.

If $X \in \mathfrak{X}_{0}\left(\mathbb{R}^{4}\right)$ is $\varphi$-reversible (resp. $\phi$-equivariant) and $\gamma(t)$ is a solution of

$$
\dot{x}=X(x)
$$

with $\gamma(0)=x_{0}$, then $\varphi \gamma(-t)$ (resp. $\left.\phi \gamma(t)\right)$ is also a solution for (2). In particular, if $X$ is a $\phi$-reversible (or $\phi$-equivariant) vector field, then the phase portrait of $X$ is symmetric with respect to the subspace $\operatorname{Fix}(\phi)$, in the sense that $\phi$ maps the phase portrait of $X$ to itself, reversing the direction of time in the reversible case.

A survey on reversible-equivariant vector fields is described in Antoneli et al. (2009), Lamb and Roberts (1996), Devaney (1976) and references therein.

In this paper, we shall restrict our study to $G$-reversible-equivariant vector fields where $G$ is finite, generated by two involutions $\{\varphi, \psi\}$ and the group homomorphism $\rho: G \rightarrow\{-1,1\}$ is given by 
$\rho(\varphi)=\rho(\psi)=-1$. In this case, by a basic group theory argument, one can prove that there is $n \geq 2$ such that $G \cong \mathbb{D}_{n}$.

Our aim is to provide an analogous form of the theorem below to the $G$-reversible-equivariant case:

THEOREM 1. Let $X \in \mathfrak{X}_{0}\left(\mathbb{R}^{2 n}\right)$ be a $\varphi$-reversible vector field, where $\varphi: \mathbb{R}^{2 n}, 0 \rightarrow \mathbb{R}^{2 n}, 0$ is a $C^{\infty}$ involution with $\operatorname{dim} \operatorname{Fix}(\varphi)=n$ as a submanifold, and let $R_{0}: \mathbb{R}^{2 n} \rightarrow \mathbb{R}^{2 n}$ be any linear involution with $\operatorname{dim} \operatorname{Fix}\left(R_{0}\right)=n$. Then there exists a $C^{\infty}$ change of coordinates $h: \mathbb{R}^{2 n}, 0 \rightarrow \mathbb{R}^{2 n}, 0$ (depending on $R_{0}$ ) such that $h_{*} X$ is $R_{0}$-reversible.

The proof of Theorem 1 is straightforward: $\varphi$ is locally conjugated to $D \varphi(0)$ by the change of coordinates $I d+d \varphi(0) \varphi$; now, $D \varphi(0)$ and $R_{0}$ are linearly conjugated (by $P$, say), as they are linear involutions with $\operatorname{dim} \operatorname{Fix}(D \varphi(0))=\operatorname{dim} \operatorname{Fix}\left(R_{0}\right)$. Now take $h=P \circ(I d+d \varphi(0) \varphi)$.

Theorem 1 is very useful when one works locally with reversible vector fields. See for example in Buzzi et al. (2009) and Teixeira (1997). It allows to always fix the involution as the following:

$$
R_{0}\left(x_{1}, \ldots, x_{2 n}\right)=\left(x_{1},-x_{2}, \ldots, x_{2 n-1},-x_{2 n}\right) .
$$

DEFINITION 2. Given a finitely generated group $G=\left\langle g_{1}, \ldots, g_{l}\right\rangle$ with the generations fixed, a representation $\sigma: G \rightarrow M_{n \times n}(\mathbb{R})$ and a vector field $X \in \mathfrak{X}_{0}\left(\mathbb{R}^{n}\right)$, we say that the representation $\sigma$ is $(X, G)$ compatible if $\sigma\left(g_{j}\right) X(x)=-X\left(\sigma\left(g_{j}\right)\right)$, for all $j=1, \ldots, l$.

We prove the following:

Theorem A: Given $X \in \mathfrak{X}_{0}^{(\alpha, \beta)}\left(\mathbb{R}^{4}\right)$, we present all the $\left(X, \mathbb{D}_{n}\right)$-compatible 4-dimensional representations of $X$, for $n=2,3,4$.

As an application of Theorem A, we obtain the following result.

THEOREM B: The Belitskii normal form for $\mathbb{D}_{4}$-reversible-equivariant vector fields in $\mathfrak{X}_{0}^{(\alpha, \beta)}\left(\mathbb{R}^{4}\right)$ is exhibited, for $\alpha, \beta$ odd integers with $(\alpha, \beta)=1$.

For further details on normal form theory, see Belitskii (2002) and Bruno (1989).

This paper is organized as follows. In Section 3 we set the problem and reduce it to a system of matricial equations. In Section 4 we prove Theorem A and in Section 5, we prove Theorem B.

\section{SETTING THE PROBLEM}

Consider $X \in \mathfrak{X}_{0}^{(\alpha, \beta)}\left(\mathbb{R}^{4}\right)$ for $\alpha \beta \neq 0$. Denote $A=D X(0)$. Let $\varphi, \psi: \mathbb{R}^{4}, 0 \rightarrow \mathbb{R}^{4}$ be involutions with $\operatorname{dim} \operatorname{Fix}(\varphi)=\operatorname{dim} \operatorname{Fix}(\psi)=2$ and suppose that $X$ is $\langle\varphi, \psi\rangle$-reversible-equivariant.

Next result will be useful in the sequel.

THEOREM 3 (Bochner and Montgomery 1946). Let $G$ be a compact group of $C^{k}$ diffeomorphisms defined on a $C^{k \geq 1}$ manifold $\mathcal{M}$. Suppose that all diffeomorphisms in $G$ have a common fixed point, say $x_{0}$. Then, there exists a $C^{k}$ coordinate system $h$ around $x_{0}$ such that all diffeomorphisms in $G$ are linear with respect to $h$. 
Putting $G=\langle\varphi, \psi\rangle$, as $\varphi(0)=0$ and $\psi(0)=0$, Theorem 3 says that there exists a coordinate system $h$ around 0 such that $\widetilde{\varphi}=h^{-1} \varphi h$ and $\widetilde{\psi}=h^{-1} \psi h$ are linear involutions. Now consider $\widetilde{X}$ as $X$ in this new system of coordinates, that is, $\tilde{X}=h_{*} X$. Then $\tilde{X}$ is $\langle\widetilde{\varphi}, \tilde{\psi}\rangle$-reversible-equivariant.

Now choose any linear involution $R_{0}: \mathbb{R}^{4} \rightarrow \mathbb{R}^{4}$ with $\operatorname{dim} \operatorname{Fix}\left(R_{0}\right)=2$. As $R_{0}$ and $\tilde{\varphi}$ are linearly conjugated, we can pass to a new system of coordinates such that $\tilde{X}$ is $\left\langle R_{0}, \widetilde{\psi}_{0}\right\rangle$-reversible-equivariant for some $\widetilde{\psi}_{0}$. However, it is not possible to choose a priori a good (linear) representative for the second involution, $\widetilde{\psi}_{0}$.

In other words, it is not possible to produce an analog version of Theorem 1 for reversible-equivariant vector fields. We shall take into account all the possible choices for the second involution.

Problem A: Let $G=\langle\varphi, \psi\rangle$ be a group generated by involutive diffeomorphisms, and $X \in \mathfrak{X}_{0}\left(\mathbb{R}^{2 n}\right)$ be a $G$-reversible-equivariant vector field. Find all of the $(X, G)$-compatible representations $\sigma$ with $\sigma(\varphi)=R_{0}, R_{0}$ given by (3).

To solve Problem A, we have to determine all the linear involutions $S$ such that $\left\langle R_{0}, S\right\rangle \cong G$ and $S D X(0)+D X(0) S=0$ (this last relation is the compatibility condition for the linear part of $X$ ).

\section{PROOF OF THEOREM A}

In this section we prove Theorem $\mathrm{A}$, that deals with $\left(X, \mathbb{D}_{n}\right)$-compatible representations for $n=2,3,4$, that is, the list of groups to be considered is: $\mathbb{D}_{2} \cong\left(\mathbb{Z}_{2} \times \mathbb{Z}_{2}\right), \mathbb{D}_{3}$ and $\mathbb{D}_{4}$.

In the rest of this section we denote by $A$ the matrix $A(\alpha, \beta)$ defined in (1).

\subsection{CASE $\mathbb{Z}_{2} \times \mathbb{Z}_{2}$}

Fix the matrix

$$
R_{0}=\left(\begin{array}{cccc}
1 & 0 & 0 & 0 \\
0 & -1 & 0 & 0 \\
0 & 0 & 1 & 0 \\
0 & 0 & 0 & -1
\end{array}\right)
$$

Note that $R_{0}^{2}=I d$ and $R_{0} A=-A R_{0}$. We need to determine all possible involutive matrices $S \in \mathbb{R}^{4 \times 4}$ such that

$$
S A=-A S
$$

and

$$
\left\langle R_{0}, S\right\rangle \cong \mathbb{Z}_{2} \times \mathbb{Z}_{2}
$$

Note that the relation $\left\langle R_{0}, S\right\rangle \cong \mathbb{Z}_{2} \times \mathbb{Z}_{2}$ is equivalent to $R_{0} S=S R_{0}$ and $S^{2}=I d$.

Put

$$
S=\left(\begin{array}{llll}
a_{1} & b_{1} & c_{1} & d_{1} \\
a_{2} & b_{2} & c_{2} & d_{2} \\
a_{3} & b_{3} & c_{3} & d_{3} \\
a_{4} & b_{4} & c_{4} & d_{4}
\end{array}\right)
$$


The relations $S A=-A S, S^{2}=I d$ and $R_{0} S=S R_{0}$ are represented by the following systems of polynomial equations:

$$
\left\{\begin{array} { l } 
{ a _ { 1 } ^ { 2 } - 1 + c _ { 1 } a _ { 3 } = 0 } \\
{ a _ { 1 } c _ { 1 } + c _ { 1 } c _ { 3 } = 0 } \\
{ b _ { 2 } d _ { 2 } + d _ { 2 } d _ { 4 } = 0 } \\
{ a _ { 3 } a _ { 1 } + c _ { 3 } a _ { 3 } = 0 } \\
{ b _ { 4 } b _ { 2 } + d _ { 4 } b _ { 4 } = 0 } \\
{ b _ { 2 } ^ { 2 } + d _ { 2 } b _ { 4 } - 1 = 0 } \\
{ c _ { 1 } a _ { 3 } + c _ { 3 } ^ { 2 } - 1 = 0 } \\
{ d _ { 2 } b _ { 4 } + d _ { 4 } ^ { 2 } - 1 = 0 }
\end{array} \quad \left\{\begin{array}{l}
-a_{1} \alpha-b_{2} \alpha=0 \\
-c_{1} \beta-\alpha d_{2}=0 \\
b_{2} \alpha+a_{1} \alpha=0 \\
d_{2} \beta+\alpha c_{1}=0 \\
-a_{3} \alpha-\beta b_{4}=0 \\
-c_{3} \beta-d_{4} \beta=0 \\
b_{4} \alpha+\beta a_{3}=0 \\
d_{4} \beta+c_{3} \beta=0
\end{array}\right.\right.
$$

Lemma 4. System (6) has 4 solutions:

$$
\begin{aligned}
S_{1}=\left(\begin{array}{cccc}
-1 & 0 & 0 & 0 \\
0 & 1 & 0 & 0 \\
0 & 0 & -1 & 0 \\
0 & 0 & 0 & 1
\end{array}\right), & S_{2}=\left(\begin{array}{cccc}
-1 & 0 & 0 & 0 \\
0 & 1 & 0 & 0 \\
0 & 0 & 1 & 0 \\
0 & 0 & 0 & -1
\end{array}\right) \\
S_{3}=\left(\begin{array}{cccc}
1 & 0 & 0 & 0 \\
0 & -1 & 0 & 0 \\
0 & 0 & -1 & 0 \\
0 & 0 & 0 & 1
\end{array}\right), & S_{4}=\left(\begin{array}{cccc}
1 & 0 & 0 & 0 \\
0 & -1 & 0 & 0 \\
0 & 0 & 1 & 0 \\
0 & 0 & 0 & -1
\end{array}\right)
\end{aligned}
$$

Proof. This can be done in Maple 12 by means of the Reduce function from the Groebner package and the usual Maple's solve function. We remark that the solution $S_{4}$ is degenerate, i.e., $S_{4}=R_{0}$. Moreover, we remark that the above representations of $\mathbb{Z}_{2} \times \mathbb{Z}_{2}$ are not equivalent.

Now we state the main result for $\mathbb{Z}_{2} \times \mathbb{Z}_{2}$-reversible vector fields. With the notation of Section 3, it assures that the linear involutions $S_{j}$ are the unique possibilities for $\widetilde{\psi_{0}}$.

THEOREM 5. Let $\Omega_{\mathbb{Z}_{2} \times \mathbb{Z}_{2}} \subset \mathfrak{X}_{0}^{(\alpha, \beta)}\left(\mathbb{R}^{4}\right)$ be the set of $\mathbb{Z}_{2} \times \mathbb{Z}_{2}$-reversible-equivariant vector fields $X \in$ $\mathfrak{X}_{0}^{(\alpha, \beta)}\left(\mathbb{R}^{4}\right)$. Then $\Omega_{\mathbb{Z}_{2} \times \mathbb{Z}_{2}}=\Omega_{1} \cup \Omega_{2} \cup \Omega_{3}$, where $X \in \Omega_{j}$ if $X$ is $\left(R_{0}, S_{j}\right)$-reversible-equivariant in some coordinate system around the origin.

PROOF. Let $X \in \Omega_{\mathbb{Z}_{2} \times \mathbb{Z}_{2}}$. Then there are distinct and nontrivial involutions $\varphi, \psi: \mathbb{R}^{4}, 0 \rightarrow \mathbb{R}^{4}, 0$ with $\varphi \psi=\psi \varphi$ such that $X$ is $\varphi$-reversible and $\psi$-reversible. By Theorem 3, there is a system of coordinates around the origin where $X$ is $R_{0}$-reversible and $S_{0}$-reversible, with $R_{0}\left(x_{1}, x_{2}, y_{1}, y_{2}\right)=\left(x_{1},-x_{2}, y_{1},-y_{2}\right)$ and $S_{0}$ a linear involution with $R_{0} S_{0}=S_{0} R_{0}$. By Lemma $4 S_{0} \in\left\{S_{1}, S_{2}, S_{3}, S_{4}\right\}$. As $\varphi \neq \psi, S_{0} \neq S_{4}$. So $X$ is $R_{0}$-reversible and $S_{j}$-reversible for some $j \in\{1,2,3\}$. Then $X \in \Omega_{1} \cup \Omega_{2} \cup \Omega_{3}$.

REMARK 6. Theorem 5 can be proved without using Lemma 4. The following technique, that also applies to Theorems 11 and 14, was communicated to us by one of the referees. 
Consider the decomposition of $\mathbb{R}^{4}$ in the generalized eigenspaces $\mathbb{R}^{4}=\operatorname{Aut}(\alpha) \oplus \operatorname{Aut}(\beta)$, where

$$
\operatorname{Aut}(\lambda)=\left\{x \in \mathbb{R}^{4} ; \exists k \geq 1:(A-\lambda \cdot I d)^{k} x=0\right\} .
$$

Then the equation $S A=-A S$ keeps the above decomposition fixed (for $\alpha \neq \beta$ ). This reduces the problem of determining $S$ to two two-dimensional linear problems, and can be easily generalized to arbitrary dimensions.

We keept the algorithmic proofs just because we are more familiar with the computational approach.

Now let us give a characterization of the vector fields which are $\left(R_{0}, S_{j}\right)$-reversible. Let us fix

$$
X(x)=A(\alpha, \beta) x+\left(f_{1}(x), f_{2}(x), f_{3}(x), f_{4}(x)\right)^{T},
$$

with $x \equiv\left(x_{1}, x_{2}, y_{1}, y_{2}\right)$. The proof of next results will be omitted.

COROLLARY 7. The vector field $(7)$ is $\left(R_{0}, S_{1}\right)$-reversible if and only if the functions $f_{j}$ satisfy

$$
\left\{\begin{array}{l}
f_{1}(x)=-f_{1}\left(x_{1},-x_{2}, y_{1},-y_{2}\right)=f_{1}\left(-x_{1}, x_{2},-y_{1}, y_{2}\right) \\
f_{2}(x)=f_{2}\left(x_{1},-x_{2}, y_{1},-y_{2}\right)=-f_{2}\left(-x_{1}, x_{2},-y_{1}, y_{2}\right) \\
f_{3}(x)=-f_{3}\left(x_{1},-x_{2}, y_{1},-y_{2}\right)=f_{3}\left(-x_{1}, x_{2},-y_{1}, y_{2}\right) \\
f_{4}(x)=f_{4}\left(x_{1},-x_{2}, y_{1},-y_{2}\right)=-f_{4}\left(-x_{1}, x_{2},-y_{1}, y_{2}\right) .
\end{array}\right.
$$

In particular, $f_{1,3}\left(x_{1}, 0, y_{1}, 0\right) \equiv 0$ and $f_{2,4}\left(0, x_{2}, 0, y_{2}\right) \equiv 0$.

COROLlARY 8. The vector field (7) is $\left(R_{0}, S_{2}\right)$-reversible if and only if the functions $f_{j}$ satisfy

$$
\left\{\begin{array}{l}
f_{1}(x)=-f_{1}\left(x_{1},-x_{2}, y_{1},-y_{2}\right)=f_{1}\left(-x_{1}, x_{2}, y_{1},-y_{2}\right) \\
f_{2}(x)=f_{2}\left(x_{1},-x_{2}, y_{1},-y_{2}\right)=-f_{2}\left(-x_{1}, x_{2}, y_{1},-y_{2}\right) \\
f_{3}(x)=-f_{3}\left(x_{1},-x_{2}, y_{1},-y_{2}\right)=-f_{3}\left(-x_{1}, x_{2}, y_{1},-y_{2}\right) \\
f_{4}(x)=f_{4}\left(x_{1},-x_{2}, y_{1},-y_{2}\right)=f_{4}\left(-x_{1}, x_{2}, y_{1},-y_{2}\right) .
\end{array}\right.
$$

In particular, $f_{1,3}\left(x_{1}, 0, y_{1}, 0\right) \equiv 0$ and $f_{2,3}\left(0, x_{2}, y_{1}, 0\right) \equiv 0$.

COROLLARY 9. The vector field $(7)$ is $\left(R_{0}, S_{3}\right)$-reversible if and only if the functions $f_{j}$ satisfy

$$
\left\{\begin{array}{l}
f_{1}(x)=-f_{1}\left(x_{1},-x_{2}, y_{1},-y_{2}\right)=-f_{1}\left(x_{1},-x_{2},-y_{1}, y_{2}\right) \\
f_{2}(x)=f_{2}\left(x_{1},-x_{2}, y_{1},-y_{2}\right)=f_{2}\left(x_{1},-x_{2},-y_{1}, y_{2}\right) \\
v f_{3}(x)=-f_{3}\left(x_{1},-x_{2}, y_{1},-y_{2}\right)=f_{3}\left(x_{1},-x_{2},-y_{1}, y_{2}\right) \\
f_{4}(x)=f_{4}\left(x_{1},-x_{2}, y_{1},-y_{2}\right)=-f_{4}\left(x_{1},-x_{2},-y_{1}, y_{2}\right) .
\end{array}\right.
$$

In particular, $f_{1,3}\left(x_{1}, 0, y_{1}, 0\right) \equiv 0$ and $f_{1,4}\left(x_{1}, 0,0, y_{2}\right) \equiv 0$. 


\subsection{CASE $\mathbb{D}_{3}$}

As above we fix the matrix

$$
R_{0}=\left(\begin{array}{cccc}
1 & 0 & 0 & 0 \\
0 & -1 & 0 & 0 \\
0 & 0 & 1 & 0 \\
0 & 0 & 0 & -1
\end{array}\right)
$$

Now we need to determine all possible involutive matrices $S \in \mathbb{R}^{4 \times 4}$ such that

$$
S A=-A S
$$

and

$$
\left\langle R_{0}, S\right\rangle \cong \mathbb{D}_{3}
$$

Considering again

$$
S=\left(\begin{array}{llll}
a_{1} & b_{1} & c_{1} & d_{1} \\
a_{2} & b_{2} & c_{2} & d_{2} \\
a_{3} & b_{3} & c_{3} & d_{3} \\
a_{4} & b_{4} & c_{4} & d_{4}
\end{array}\right),
$$

the equations $S A+A S=0, S^{2}-I d=0$ and $\left(R_{0} S\right)^{3}-I d=0$ are equivalent to a huge system of equations. Their expression will be not presented.

LEMMA 10. The system generated by the above conditions has the following non degenerate solutions:

$$
S_{1}=\left(\begin{array}{cccc}
-\frac{1}{2} & \frac{\sqrt{3}}{2} & 0 & 0 \\
\frac{\sqrt{3}}{2} & \frac{1}{2} & 0 & 0 \\
0 & 0 & -\frac{1}{2} & \frac{\sqrt{3}}{2} \\
0 & 0 & \frac{\sqrt{3}}{2} & \frac{1}{2}
\end{array}\right), S_{2}=\left(\begin{array}{cccc}
-\frac{1}{2} & \frac{\sqrt{3}}{2} & 0 & 0 \\
\frac{\sqrt{3}}{2} & \frac{1}{2} & 0 & 0 \\
0 & 0 & 1 & 0 \\
0 & 0 & 0 & -1
\end{array}\right), S_{3}=\left(\begin{array}{cccc}
1 & 0 & 0 & 0 \\
0 & -1 & 0 & 0 \\
0 & 0 & -\frac{1}{2} & \frac{\sqrt{3}}{2} \\
0 & 0 & \frac{\sqrt{3}}{2} & \frac{1}{2}
\end{array}\right) .
$$

Proof. Again, the proof can be done in Maple 12 using the Reduce function from the Groebner package and the usual Maple's solve function.

At this point, we can state the following:

THEOREM 11. Let $\Omega_{\mathbb{D}_{3}} \subset \mathfrak{X}_{0}^{(\alpha, \beta)}\left(\mathbb{R}^{4}\right)$ be the set of $\mathbb{D}_{3}$-reversible-equivariant vector fields $X \in$ $\mathfrak{X}_{0}^{(\alpha, \beta)}\left(\mathbb{R}^{4}\right)$. Then $\Omega_{\mathbb{D}_{3}}=\Omega_{1} \cup \Omega_{2} \cup \Omega_{3}$, where $X \in \Omega_{j}$ if $X$ is $\left(R_{0}, S_{j}\right)$-reversible-equivariant in some coordinate system around the origin.

PROOF. This proof is very similar to that of Theorem 5 .

Now we present some results in the sense of Corollary 7 applied to $\mathbb{D}_{3}$-reversible vector fields. The characterization is given just for the $\left(R_{0}, S_{2}\right)$-reversible vector fields. Similar statements for $\left(R_{0}, S_{j}\right)$ reversible vector fields, with $j \in\{1,3\}$ can be easily deduced. 
Let us fix again

$$
X(x)=A(\alpha, \beta) x+\left(g_{1}(x), g_{2}(x), g_{3}(x), g_{4}(x)\right)^{T},
$$

with $x \equiv\left(x_{1}, x_{2}, y_{1}, y_{2}\right)$. Keeping the same notation of Section 4.1 , we have now $\left\langle R_{0}, S_{j}\right\rangle \cong \mathbb{D}_{3}$. We consider for instance $j=2$.

COROLLARY 12. The vector field $(12)$ is $\left(R_{0}, S_{2}\right)$-reversible if and only if the functions $g_{j}$ satisfy

$$
\left\{\begin{array}{l}
g_{1}(x)=-g_{1}\left(x_{1},-x_{2}, y_{1},-y_{2}\right) \\
g_{2}(x)=g_{2}\left(x_{1},-x_{2}, y_{1},-y_{2}\right) \\
g_{3}(x)=-g_{3}\left(x_{1},-x_{2}, y_{1},-y_{2}\right) \\
g_{4}(x)=g_{4}\left(x_{1},-x_{2}, y_{1},-y_{2}\right)
\end{array}\right.
$$

and

$$
\left\{\begin{array}{l}
g_{1}(x)=-g_{1}\left(x_{1},-x_{2},-\frac{1}{2} y_{1}+\frac{\sqrt{3}}{2} y_{2}, \frac{\sqrt{3}}{2} y_{1}+\frac{1}{2} y_{2}\right) \\
g_{2}(x)=g_{2}\left(x_{1},-x_{2},-\frac{1}{2} y_{1}+\frac{\sqrt{3}}{2} y_{2}, \frac{\sqrt{3}}{2} y_{1}+\frac{1}{2} y_{2}\right) \\
\frac{1}{2} g_{3}(x)-\frac{\sqrt{3}}{2} g_{4}(x)=g_{3}\left(x_{1},-x_{2},-\frac{1}{2} y_{1}+\frac{\sqrt{3}}{2} y_{2}, \frac{\sqrt{3}}{2} y_{1}+\frac{1}{2} y_{2}\right) \\
\frac{\sqrt{3}}{2} g_{3}(x)+\frac{1}{2} g_{4}(x)=-g_{4}\left(x_{1},-x_{2},-\frac{1}{2} y_{1}+\frac{\sqrt{3}}{2} y_{2}, \frac{\sqrt{3}}{2} y_{1}+\frac{1}{2} y_{2}\right)
\end{array}\right.
$$

In particular $g_{1,3}\left(x_{1}, 0, y_{1}, 0\right) \equiv 0$ and $g_{2,4}\left(0, x_{2}, 0, y_{2}\right) \equiv 0$.

The next section deals with the characterization of the $\mathbb{D}_{4}$-reversible vector fields. The analysis of the $\mathbb{D}_{3}$-reversible case will be omitted since it is very similar to the $\mathbb{D}_{4}$-reversible case and this last case is more interesting (there are more representations).

\subsection{CASE $\mathbb{D}_{4}$}

Fix the matrix

$$
R_{0}=\left(\begin{array}{cccc}
1 & 0 & 0 & 0 \\
0 & -1 & 0 & 0 \\
0 & 0 & 1 & 0 \\
0 & 0 & 0 & -1
\end{array}\right)
$$

Again, our aim is to determine all the possible involutive matrices $S \in \mathbb{R}^{4 \times 4}$ such that

$$
S A=-A S
$$

and

$$
\left\langle R_{0}, S\right\rangle \cong \mathbb{D}_{4}
$$

Considering again

$$
S=\left(\begin{array}{llll}
a_{1} & b_{1} & c_{1} & d_{1} \\
a_{2} & b_{2} & c_{2} & d_{2} \\
a_{3} & b_{3} & c_{3} & d_{3} \\
a_{4} & b_{4} & c_{4} & d_{4}
\end{array}\right)
$$


the equations $S A+A S=0, S^{2}-I d=0$ and $\left(R_{0} S\right)^{4}-I d=0$ are represented by a easily deduced but huge system having 12 non degenerate solutions, arranged in the following way:

$$
\begin{aligned}
& \Xi_{1}=\left\{\left(\begin{array}{cccc}
0 & -1 & 0 & 0 \\
-1 & 0 & 0 & 0 \\
0 & 0 & 1 & 0 \\
0 & 0 & 0 & -1
\end{array}\right),\left(\begin{array}{cccc}
0 & 1 & 0 & 0 \\
1 & 0 & 0 & 0 \\
0 & 0 & 1 & 0 \\
0 & 0 & 0 & -1
\end{array}\right)\right\}, \quad \Xi_{2}=\left\{\left(\begin{array}{cccc}
-1 & 0 & 0 & 0 \\
0 & 1 & 0 & 0 \\
0 & 0 & 0 & 1 \\
0 & 0 & 1 & 0
\end{array}\right),\left(\begin{array}{cccc}
-1 & 0 & 0 & 0 \\
0 & 1 & 0 & 0 \\
0 & 0 & 0 & -1 \\
0 & 0 & -1 & 0
\end{array}\right)\right\}
\end{aligned}
$$

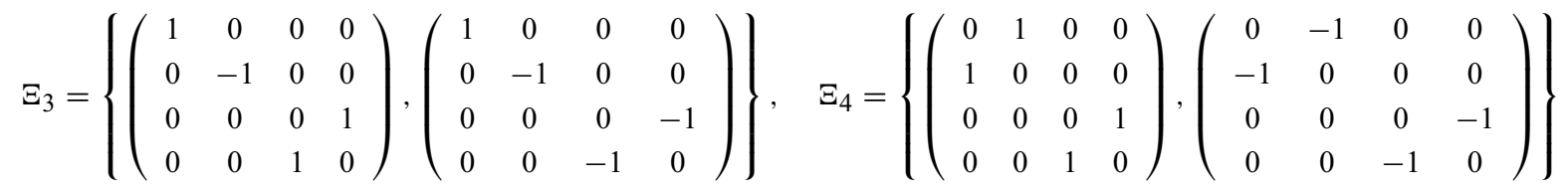

$$
\begin{aligned}
& \Xi_{5}=\left\{\left(\begin{array}{cccc}
0 & 1 & 0 & 0 \\
1 & 0 & 0 & 0 \\
0 & 0 & -1 & 0 \\
0 & 0 & 0 & 1
\end{array}\right),\left(\begin{array}{cccc}
0 & -1 & 0 & 0 \\
-1 & 0 & 0 & 0 \\
0 & 0 & -1 & 0 \\
0 & 0 & 0 & 1
\end{array}\right)\right\}, \quad \Xi_{6}=\left\{\left(\begin{array}{cccc}
0 & 1 & 0 & 0 \\
1 & 0 & 0 & 0 \\
0 & 0 & 0 & -1 \\
0 & 0 & -1 & 0
\end{array}\right),\left(\begin{array}{cccc}
0 & -1 & 0 & 0 \\
-1 & 0 & 0 & 0 \\
0 & 0 & 0 & 1 \\
0 & 0 & 1 & 0
\end{array}\right)\right\}
\end{aligned}
$$

Recall that the above arrangement has obeyed the rule:

Lemma 13. $S_{i}, S_{j} \in \Xi_{k} \Leftrightarrow\left\langle R_{0}, S_{i}\right\rangle=\left\langle R_{0}, S_{j}\right\rangle$.

For each $i \in\{1, \ldots, 6\}$, denote by $S_{i}$ one of the elements of $\Xi_{i}$. The proof of the next result follows immediately the above lemmas.

THEOREM 14. Let $\Omega_{\mathbb{D}_{4}} \subset \mathfrak{X}_{0}^{(\alpha, \beta)}\left(\mathbb{R}^{4}\right)$ be the set of $\mathbb{D}_{4}$-reversible-equivariant vector fields $X \in$ $\mathfrak{X}_{0}^{(\alpha, \beta)}\left(\mathbb{R}^{4}\right)$. Then $\Omega_{\mathbb{D}_{4}}=\Omega_{1} \cup \Omega_{2} \cup \ldots \cup \Omega_{6}$, where $X \in \Omega_{j}$ if $X$ is $\left(R_{0}, S_{j}\right)$-reversible-equivariant in some coordinate system around the origin.

Proof. This proof is very similar to that of Theorem 5. It will be omitted.

Now we present some results in the sense of Corollary 7 applied to $\mathbb{D}_{4}$-reversible vector fields. The characterization is given just for the $\left(R_{0}, S_{1}\right)$-reversible vector fields. Similar statements for $\left(R_{0}, S_{j}\right)$ reversible vector fields, with $j \in\{2,3,4,5,6\}$ can be easily deduced.

Let us fix again

$$
X(x)=A(\alpha, \beta) x+\left(g_{1}(x), g_{2}(x), g_{3}(x), g_{4}(x)\right)^{T},
$$

with $x \equiv\left(x_{1}, x_{2}, y_{1}, y_{2}\right)$. Keeping the same notation of Section 4.1 , we have now $\left\langle R_{0}, S_{j}\right\rangle \cong \mathbb{D}_{4}$.

COROLLARY 15. The vector field $(15)$ is $\left(R_{0}, S_{1}\right)$-reversible if and only if the functions $g_{j}$ satisfy

$$
\left\{\begin{array}{l}
g_{1}(x)=-g_{1}\left(x_{1},-x_{2}, y_{1},-y_{2}\right)=-g_{2}\left(x_{2}, x_{1}, y_{1},-y_{2}\right) \\
g_{2}(x)=g_{2}\left(x_{1},-x_{2}, y_{1},-y_{2}\right)=-g_{1}\left(x_{2}, x_{1}, y_{1},-y_{2}\right) \\
g_{3}(x)=-g_{3}\left(x_{1},-x_{2}, y_{1},-y_{2}\right)=-g_{3}\left(x_{2}, x_{1}, y_{1},-y_{2}\right) \\
g_{4}(x)=g_{4}\left(x_{1},-x_{2}, y_{1},-y_{2}\right)=g_{4}\left(x_{2}, x_{1}, y_{1},-y_{2}\right)
\end{array}\right.
$$

In particular $g_{1,3}\left(x_{1}, 0, y_{1}, 0\right) \equiv 0$ and $g_{2,4}\left(0, x_{2}, 0, y_{2}\right) \equiv 0$ 


\section{APPLICATIONS TO NORMAL FORMS (PROOF OF THEOREM B)}

Let $X \in \mathfrak{X}_{0}^{(\alpha, \beta)}\left(\mathbb{R}^{4}\right)$ be a $\mathbb{D}_{4}$-reversible vector field and $X^{N}$ its reversible-equivariant Belitskii normal form.

To compute the expression of $X^{N}$, we have to consider the following possibilities of the parameter $\lambda=\alpha \beta^{-1}$ :

(i) $\lambda \notin \mathbb{Q}$,

(ii) $\lambda=1$,

(iii) $\lambda=p q^{-1} \neq 1$, with $p, q$ integers and $(p, q)=1$.

In the case (i), one can show that the normal forms for the reversible and reversible-equivariant cases are essentially the same. This means that any reversible field with such linear approximation is automatically reversible-equivariant. In view of this, case (i) is not interesting, and its analysis will be omitted. We just note that case (ii) will not be discussed here because of its deep degeneracy, as the range of its homological operator

$$
L_{A(\alpha, \alpha)}: H^{k} \rightarrow H^{k}
$$

is a very low dimensional subspace of $H^{k}$. Also recall that we suppose $\alpha \neq \beta$ in the definition of $A(\alpha, \beta)$.

Our goal is to focus on the case (iii). Put $\alpha=p$ and $\beta=q$, with $p, q \in \mathbb{Z}$ and $(p, q)=1$. How to compute a normal form which applies for all $\mathbb{D}_{4}$-reversible vector fields, without choosing specific involutions?

According to the results in the last section, it suffices to show that $X^{N}$ satisfies

$$
R_{0}\left(X^{N}(x)\right)=-X^{N}\left(R_{0}(x)\right)
$$

and

$$
S_{j}\left(X^{N}(x)\right)=-X^{N}\left(S_{j}(x)\right), j=1, \ldots, 6,
$$

with $S_{j}$ given on Lemma 13, as the fixed choice for the representative of $\Xi_{i}$.

First of all, we consider complex coordinates $\left(z_{1}, z_{2}\right) \in \mathbb{C}^{2}$ instead of $\left(x_{1}, x_{2}, y_{1}, y_{2}\right) \in \mathbb{R}^{4}$ :

$$
\left\{\begin{array}{l}
z_{1}=x_{1}+i x_{2} \\
z_{2}=y_{1}+i y_{2}
\end{array}\right.
$$

We will write $\Re(z)$ for the real part of the complex number $z$ and $\Im(z)$ for its imaginary part.

Define

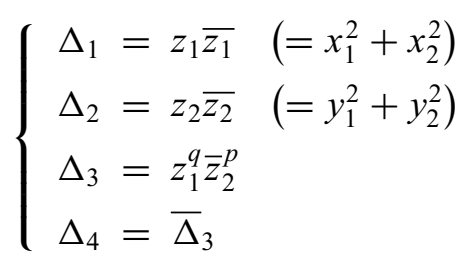

Note that each $\Delta_{j}$ corresponds to a resonance relation among the eigenvalues of the matrix $A(p, q)$ (given in (1)). For instance, if $\lambda_{1}=p i$ and $\lambda_{2}=q i$, then

$$
m \lambda_{1}+m \overline{\lambda_{1}}+n \lambda_{2}+n \overline{\lambda_{2}}=0
$$

for all $m, n \geq 1$. Then

$$
\lambda_{1}=(m+1) \lambda_{1}+m \overline{\lambda_{1}}+n \lambda_{2}+n \overline{\lambda_{2}}
$$


is a resonance relation, and this relation corresponds to the resonant monomial

$$
z_{1}\left(z_{1} \bar{z}_{1}\right)^{m}\left(z_{2} \bar{z}_{2}\right)^{m} \frac{\partial}{\partial z_{1}}=z_{1} \Delta_{1}^{m} \Delta_{2}^{m} \frac{\partial}{\partial z_{1}} .
$$

Collecting all the resonant monomials, the complex Belitskii normal form for $X$ in this case is expressed by

$$
\left\{\begin{array}{l}
\dot{z}_{1}=p i z_{1}+z_{1} f_{1}\left(\Delta_{1}, \Delta_{2}, \Delta_{3}, \Delta_{4}\right)+\bar{z}_{1}^{q-1} z_{2}^{p} f_{2}\left(\Delta_{1}, \Delta_{2}, \Delta_{3}, \Delta_{4}\right) \\
\dot{z}_{2}=q i z_{2}+z_{2} g_{1}\left(\Delta_{1}, \Delta_{2}, \Delta_{3}, \Delta_{4}\right)+z_{1}^{q} \bar{z}_{2}^{p-1} g_{2}\left(\Delta_{1}, \Delta_{2}, \Delta_{3}, \Delta_{4}\right),
\end{array}\right.
$$

with $f_{j}, g_{j}$ without linear and constant terms. For more details on the construction of this Belitskii normal form, we refer to Belitskii (2002).

Now we consider the effects of $\mathbb{D}_{4}$-reversibility on the system (18). Writing our involutions in complex coordinates, we derive immediately that

LEMMA 16. Let

$$
\begin{aligned}
\varphi_{0}\left(z_{1}, z_{2}\right) & =-\left(\overline{z_{1}}, \overline{z_{2}}\right) & & \\
\varphi_{1}\left(z_{1}, z_{2}\right) & =\left(i \overline{z_{1}}, \overline{z_{2}}\right) & \varphi_{2}\left(z_{1}, z_{2}\right) & =-\left(\overline{z_{1}}, \overline{i z_{2}}\right) \\
\varphi_{3}\left(z_{1}, z_{2}\right) & =\left(\overline{z_{1}},-\overline{i z_{2}}\right) & \varphi_{4}\left(z_{1}, z_{2}\right) & =-\left(\overline{i z_{1}}, \overline{i z_{2}}\right) \\
\varphi_{5}\left(z_{1}, z_{2}\right) & =-\left(\overline{i z_{1}}, \overline{z_{2}}\right) & \varphi_{6}\left(z_{1}, z_{2}\right) & =\left(-\overline{i z_{1}}, \overline{i z_{2}}\right)
\end{aligned}
$$

Then each group $\left\langle\varphi_{0}, \varphi_{j}\right\rangle$ corresponds to $\left\langle R_{0}, S_{j}\right\rangle, j=1, \ldots, 6$.

To compute a $\mathbb{D}_{4}$-reversible normal form for a vector field, one has first to define which of the groups in Lemma 16 can be used to do the calculations. Now we establish a normal form of a $\mathbb{D}_{4}$-reversible and $p: q$-resonant vector field $X$, depending only on $p, q$ and not on the involutions generating $\mathbb{D}_{4}$ :

THEOREM 17. Let $p, q$ be odd numbers with $p q>1$ and $X \in \mathfrak{X}_{0}^{(p, q)}\left(\mathbb{R}^{4}\right)$ be a $\mathbb{D}_{4}$-reversible vector field. Then $X$ is formally conjugated to the following system:

$$
\left\{\begin{array}{l}
\dot{x_{1}}=-p x_{2}-x_{2} \sum_{i+j=1}^{\infty} a_{i j} \Delta_{1}^{i} \Delta_{2}^{j} \\
\dot{x_{2}}=p x_{1}+x_{1} \sum_{i+j=1}^{\infty} a_{i j} \Delta_{1}^{i} \Delta_{2}^{j} \\
\dot{y_{1}}=-q y_{2}-y_{2} \sum_{i+j=1}^{\infty} b_{i j} \Delta_{1}^{i} \Delta_{2}^{j} \\
\dot{y_{2}}=q y_{1}+y_{1} \sum_{i+j=1}^{\infty} b_{i j} \Delta_{1}^{i} \Delta_{2}^{j},
\end{array}\right.
$$

with $a_{i j}, b_{i j} \in \mathbb{R}$ depending on $j^{k} X(0)$, for $k=i+j$.

REMARK 18. The hypothesis on $p, q$ given in Theorem 17 can be relaxed. In fact, if $p, q$ satisfies the following conditions

$$
\left\{\begin{array}{l}
q \equiv_{4} 1 \text { or } q \equiv_{4} 3 \text { or }\left(q \equiv_{4} 0 \text { and } p+q=2 k+1\right) \text { or }\left(q \equiv_{4} 2 \text { and } p+q=2 k\right) \\
p \equiv_{4} 1 \text { or } p \equiv_{4} 2 \text { or } p \equiv_{4} 3 \\
p \equiv_{4} 1 \text { or } p \equiv_{4} 3 \text { or }\left(p \equiv_{4} 0 \text { and } q=2 k+1\right) \text { or }\left(p \equiv_{4} 2 \text { and } q=2 k\right)
\end{array}\right.
$$

then the conclusions of Theorem 17 are also valid (see Martins 2008).

REMARK 19. The normal form (19) coincides (in the nonlinear terms) with the normal form of a reversible vector field $X \in \mathfrak{X}_{0}^{(\alpha, \beta)}\left(\mathbb{R}^{4}\right)$ with $\alpha \beta^{-1} \notin \mathbb{Q}$. Remember that this fact allowed us to discard the case $\alpha \beta^{-1} \notin \mathbb{Q}$ at the beginning of this section. 
The proof of Theorem 17 (even with the hypothesis of Remark 18) is based on a sequence of lemmas. The idea is just to show that with some hypothesis on $p$ and $q$, all the coefficients of $\Delta_{3}$ and $\Delta_{4}$ in the reversible-equivariant analogue of (18) must be zero.

First let us focus on the monomials that are never killed by the reversible-equivariant structure.

LEMMA 20. Let $v=a z_{j} \Delta_{1}^{m} \Delta_{2}^{n} \frac{\partial}{\partial z_{j}}$, $a \in \mathbb{C}$. So, for any $j \in\{1, \ldots, 6\}$, the $\varphi_{j}$-reversibility implies $\bar{a}=-a($ or $\Re(a)=0)$. In particular, these terms are always present (generically) in the normal form.

PROOF. From

$$
\varphi_{0}\left(a z_{1} \Delta_{1}^{m} \Delta_{2}^{n} \frac{\partial}{\partial z_{1}}\right)=-\bar{a} \overline{z_{1}} \Delta_{1}^{m} \Delta_{2}^{n} \frac{\partial}{\partial z_{1}}
$$

and

$$
\left.a z_{1} \Delta_{1}^{m} \Delta_{2}^{n} \frac{\partial}{\partial z_{1}}\right|_{\left(-\overline{z_{1}},-\overline{z_{2}}\right)}=-a \overline{z_{1}} \Delta_{1}^{m} \Delta_{2}^{n} \frac{\partial}{\partial z_{1}}
$$

follows that $-\bar{a}=a$.

Now let us see what happens with the monomials of type $\left(\overline{z_{1}}\right)^{q-1} z_{2}^{p} \frac{\partial}{\partial z_{1}}$. We mention that only for such monomials a complete proof will be presented. The other cases are similar. Moreover, we will give the statement and the proof as stated in Remark 18.

\begin{tabular}{|c|c|c|}
\hline reversibility & hypothesis on $p, q$ & conditions on $b$ \\
\hline \multirow{2}{*}{$\varphi_{0}$} & $p+q$ even & $\Re(b)=0$ \\
\hline & $p+q$ odd & $\Im(b)=0$ \\
\hline \multirow[t]{4}{*}{$\varphi_{1}$} & $q \equiv_{4} 0$ & $\Re(b)=0$ \\
\hline & $q \equiv_{4} 1$ & $\Re(b)=-\Im(b)$ \\
\hline & $q \equiv_{4} 2$ & $\Im(b)=0$ \\
\hline & $q \equiv_{4} 3$ & $\Re(b)=\Im(b)$ \\
\hline \multirow[t]{8}{*}{$\varphi_{2}$} & $p \equiv_{4} 0, q$ even & $\Re(b)=0$ \\
\hline & $p \equiv_{4} 0, q$ odd & $\Im(b)=0$ \\
\hline & $q \equiv_{4} 1, q$ even & $\Re(b)=\Im(b)$ \\
\hline & $q \equiv_{4} 1, q$ odd & $\Re(b)=-\Im(b)$ \\
\hline & $q \equiv_{4} 2, q$ even & $\Im(b)=0$ \\
\hline & $q \equiv_{4} 2, q$ odd & $\Re(b)=0$ \\
\hline & $q \equiv_{4} 3, q$ even & $\Re(b)=-\Im(b)$ \\
\hline & $q \equiv_{4} 3, q$ odd & $\Re(b)=\Im(b)$ \\
\hline \multirow[t]{4}{*}{$\varphi_{3}$} & $p \equiv_{4} 0$ & $\mathfrak{R}(b)=0$ \\
\hline & $p \equiv_{4} 1$ & $\mathfrak{R}(b)=\Im(b)$ \\
\hline & $p \equiv_{4} 2$ & $\Im(b)=0$ \\
\hline & $p \equiv_{4} 3$ & $\Re(b)=-\Im(b)$ \\
\hline
\end{tabular}

Lemma 21. Let $v=b{\overline{z_{1}}}^{q-1} z_{2}^{p} \frac{\partial}{\partial z_{1}}, b \in \mathbb{C}$. So, we establish the following tables: 


\begin{tabular}{c|l|l} 
reversibility & hypothesis on $p, q$ & conditions on $b$ \\
\hline$\varphi_{4}$ & $p+q \equiv_{4} 0, q$ even & $\Re(b)=0$ \\
\hline & $p+q \equiv_{4} 0, q$ odd & $\Im(b)=0$ \\
\hline & $p+q \equiv_{4} 1, q$ even & $\Re(b)=\Im(b)$ \\
\hline & $p+q \equiv_{4} 1, q$ odd & $\Re(b)=-\Im(b)$ \\
\hline & $p+q \equiv_{4} 2, q$ even & $\Im(b)=0$ \\
\hline & $p+q \equiv_{4} 2, q$ odd & $\Re(b)=0$ \\
\hline & $p+q \equiv_{4} 3, q$ even & $\Re(b)=-\Im(b)$ \\
\hline & $p+q \equiv_{4} 3, q$ odd & $\Im(b)=\Im(b)$ \\
\hline & $q \equiv_{4} 0, p+q$ even & $\Re(b)=0$ \\
\hline & $q \equiv_{4} 0, p+q$ odd & $\Im(b)=0$ \\
\hline & $q \equiv_{4} 1, p+q$ even & $\Re(b)=\Im(b)$ \\
\hline & $q \equiv_{4} 1, p+q$ odd & $\Re(b)=-\Im(b)$ \\
\hline & $q \equiv_{4} 2, p+q$ even & $\Im(b)=0$ \\
\hline & $q \equiv_{4} 2, p+q$ odd & $\Re(b)=0$ \\
\hline & $q \equiv_{4} 3, p+q$ even & $\Re(b)=-\Im(b)$ \\
\hline & $q \equiv_{4} 3, p+q$ odd & $\Re(b)=\Im(b)$ \\
\hline & $p+q \equiv_{4} 0$ & $\Re(b)=0$ \\
\hline & $p+q \equiv_{4} 1$ & $\Re(b)=-\Im(b)$ \\
\hline & $p+q \equiv_{4} 2$ & $\Im(b)=0$ \\
\hline & $p+q \equiv_{4} 3$ & $\Re(b)=\Im(b)$ \\
\hline & &
\end{tabular}

PROOF. Let us give the proof for $\varphi_{2}$-reversibility. The proof of any other case is similar. Note that

$$
\left\{\begin{array}{l}
\varphi_{2}\left(v\left(z_{1}, z_{2}\right)\right)=-\bar{b} z_{1}^{q-1}{\overline{z_{2}}}^{p} \frac{\partial}{\partial z_{1}} \\
v\left(\varphi_{2}\left(z_{1}, z_{2}\right)\right)=b(-1)^{q-1} i^{p} z_{1}^{q-1}{\overline{z_{2}}}^{p}
\end{array}\right.
$$

Then, from $\varphi_{2}(v(z))=-v\left(\varphi_{2}(z)\right)$ we have $\bar{b}=(-1)^{q-1} i^{p} b$. Now we apply the hypotheses on $p, q$ and the proof follows in a straightforward way.

Next corollary is the first of a sequence of results establishing that some monomial do not appear in the normal form:

COROLlaRY 22. Let $X \in \mathfrak{X}_{0}^{(p, q)}\left(\mathbb{R}^{4}\right)$ be a $\left\langle\varphi_{0}, \varphi_{j}\right\rangle$-reversible vector field. Then if

- $q \equiv 1 \bmod 4$ or

- $q \equiv 3 \bmod 4$ or

- $q \equiv 0 \bmod 4$ and $p+q$ odd or

- $q \equiv 2 \bmod 4$ and $p+q$ even,

then the normal form of $X$ does not contain monomials of the form

$$
a_{1}{\overline{z_{1}}}^{n q-1} z_{2}^{n} p \frac{\partial}{\partial z_{1}}, \quad a_{2} z_{1}^{m q} \bar{z}_{2}^{m p-1} \frac{\partial}{\partial z_{2}}, a_{1}, a_{2} \in \mathbb{C} .
$$


Proof. Observe that the $\varphi_{0}, \varphi_{j}$-reversibility implies that the coefficients in (20) satisfy

$$
\Re\left(a_{j}\right)=\Im\left(a_{j}\right)=0 .
$$

REMARK 23. Note that if $p, q$ are odd with $p q>1$, then they satisfy the hypothesis of Corollary 22 .

The following results can be proved in a similar way as we have done in Lemma 21 and Corollary 22. Proposition 24. Let $X \in \mathfrak{X}_{0}^{(p, q)}\left(\mathbb{R}^{4}\right)$ be a $\left\langle\varphi_{0}, \varphi_{j}\right\rangle$-reversible vector field. If one of the following conditions is satisfied:

(i) $q \equiv 1 \bmod 4$,

(ii) $q \equiv 3 \bmod 4$,

(iii) $q \equiv 0 \bmod 4$ and $p+q$

(iv) $q \equiv 2 \bmod 4$ and $p+q$ even,

then the normal form of $X$, given in (18), does not have monomials of type

$$
z_{1}\left(z_{1}^{q} \bar{z}_{2}^{p}\right)^{m} \frac{\partial}{\partial z_{1}}, \quad z_{2}\left(z_{1}^{q} \bar{z}_{2}^{p}\right)^{m} \frac{\partial}{\partial z_{2}}, \quad m \geq 1
$$

Proposition 25. Let $X \in \mathfrak{X}_{0}^{(p, q)}\left(\mathbb{R}^{4}\right)$ be a $\left\langle\varphi_{0}, \varphi_{j}\right\rangle$-reversible vector field. If one of the following conditions is satisfied

(i) $q \equiv 1 \bmod 4$,

(ii) $q \equiv 3 \bmod 4$,

(iii) $q \equiv 0 \bmod 4$ and $p+q$ odd,

(iv) $q \equiv 2 \bmod 4$ and $p+q$ even,

then the normal form of $X$, given in (18), does not have monomials of type

$$
z_{1}\left(\overline{z_{1}^{q} \bar{z}_{2}^{p}}\right)^{m} \frac{\partial}{\partial z_{1}}, \quad z_{2}\left(\overline{z_{1}^{q} \bar{z}_{2}^{p}}\right)^{m} \frac{\partial}{\partial z_{2}}, \quad m \geq 1
$$

REMARK 26. In fact, the conditions imposed on $\lambda$ in the last results are used just to assure the $\left\langle\varphi_{0}, \varphi_{j}\right\rangle$ reversibility of the vector field $X$ with $j=1$. For $2 \leq j \leq 6$, the normal form only contains monomials of type

$$
z_{j} \Delta_{1}^{m} \Delta_{2}^{n} \frac{\partial}{\partial z_{j}}
$$

Now, to prove Theorem 17, we have just to combine all lemmas, corollaries and propositions given above.

Proof of TheOREM 17. Note that the conditions imposed on $\lambda$ in Theorem 17 fit into the hypothesis of Corollary 22 and Propositions 24 and 25. So, if $p, q$ are odd numbers with $p q>1$, then the normal form just have monomials of type

$$
z_{j} \Delta_{1}^{m} \Delta_{2}^{n} \frac{\partial}{\partial z_{j}}, j=1,2, m, n>1
$$




\section{CONCLUSIONS}

In Theorem A we have classified all involutions that make a vector field $X \in \mathfrak{X}_{0}^{(p, q)}\left(\mathbb{R}^{4}\right)\langle\varphi, \psi\rangle$-reversible when the order of the group $\langle\varphi, \psi\rangle$ is smaller than 9 .

We used the classification obtained in Theorem A to simplify the Belitskii normal form of $\mathbb{D}_{4}$-reversible vector fields in $\mathbb{R}^{4}$, according to their resonances.

A normal form can be used to study stability questions and also reveal hidden symmetries. It also paves the way to get first integrals and sometimes to show that the system is an integrable hamiltonian system. Moreover, the truncated normal form gives a good approximation (or at least an asymptotic one) for the original vector field. So it is very important to write the normal form as simply as possible.

Our results show that in some cases it is possible to write the normal form of $\mathbb{D}_{4}$-reversible vector fields near a resonant singularity in the simplest possible way, that is, without the resonant terms coming from the nontrivial resonant relation among the eigenvalues. This allows us to write, for instance, the center manifold reduction in the simplest possible way.

We remark that the same approach can be made to the discrete version of the problem, or when the singularity is not elliptic (see for example Jacquemard and Teixeira 2002).

One can easily generalize the results presented here mainly in two directions: for vector fields on higher dimensional spaces and for groups with higher order. In both cases the hard missions are to face the normal form calculations and to solve some very complicated system of algebraic equations.

\section{ACKNOWLEDGMENTS}

This research was partially supported by Conselho Nacional de Desenvolvimento Científico e Tecnológico (CNPq) Brazil process No. 134619/2006-4 (Martins) and by Fundação de Amparo à Pesquisa do Estado de São Paulo (FAPESP) Brazil projects numbers 2007/05215-4 (Martins) and 2007/06896-5 (Teixeira). The authors want to thank the referees for many helpful comments and suggestions.

\section{RESUMO}

Este artigo utiliza ferramentas da teoria de grupos e computação simbólica para dar uma classificação das representações de grupos finitos de ordem menor ou igual a 9 que podem ser consideradas no estudo local de campos vetoriais reversíveis-equivariantes em $\mathbb{R}^{4}$. Os resultados são obtidos resolvendo algebricamente equações matriciais. Em particular, exibimos as involuções utilizadas no estudo local de campos vetoriais reversíveis-equivariantes. Baseado em tal abordagem, nós apresentamos, para cada elemento desta classe, uma forma normal de Belitskii simplificada.

Palavras-chave: Sistemas dinâmicos reversíveis-equivariantes, simetrias involutórias, formas normais.

\section{REFERENCES}

ANtONeli F ET AL. 2009. Invariant theory and reversible-equivariant vector fields. J Pure Appl Algebra 213: 649-663.

BeLitSKII G. 2002. $C^{\infty}$-normal forms of local vector fields. Symmetry and perturbation theory. Acta Appl Math 70: 23-41.

BIRKhoff GD. 1915. The restricted problem of three bodies. Rend Circ Mat Palermo 39: 265-334. 
Bochner S And Montgomery D. 1946. Locally Compact Groups of Differentiable Transformations. Ann of Math (2)47: 639-653.

BRUNO AD. 1989. Local methods in nonlinear differential equations. Part I. The local method of nonlinear analysis of differential equations. Part II. The sets of analyticity of a normalizing transformation. Springer Series in Soviet Mathematics. Springer-Verlag, Berlin, $\mathrm{x}+348$ p.

Buzzi CA, Llibre J ANd Medrado JCR. 2009. Phase portraits of reversible linear differential systems with cubic homogeneous polynomial nonlinearities having a non-degenerate center at the origin. Qual Theory Dyn Syst 7: 369-403.

DeVaney R. 1976. Reversible Diffeomorphisms and Flows. Trans Amer Math Soc 218: 89-113.

JACQUEMARD A AND TEIXEIRA MA. 2002. Effective algebraic geometry and normal forms of reversible mappings. Rev Mat Complut 15: 31-55.

KNUS M-A ET AL. 1998. The book of involutions. American Mathematical Society, Providence, RI, USA.

LAmB JSW And RoberTs JAG. 1996. Time-Reversal Symmetry in Dynamical Systems: A Survey. Time-reversal symmetry in dynamical systems. Coventry, UK. Phys D 112: 1-39.

MARTINS RM. 2008. A estrutura Hamiltoniana dos campos reversíveis em 4D. Master Thesis, Universidade Estadual de Campinas, UNICAMP, Campinas, SP, Brasil. (Unpublished).

TeIXeIRA MA. 1997. Singularities of reversible vector fields. Phys D 100: 101-118. 